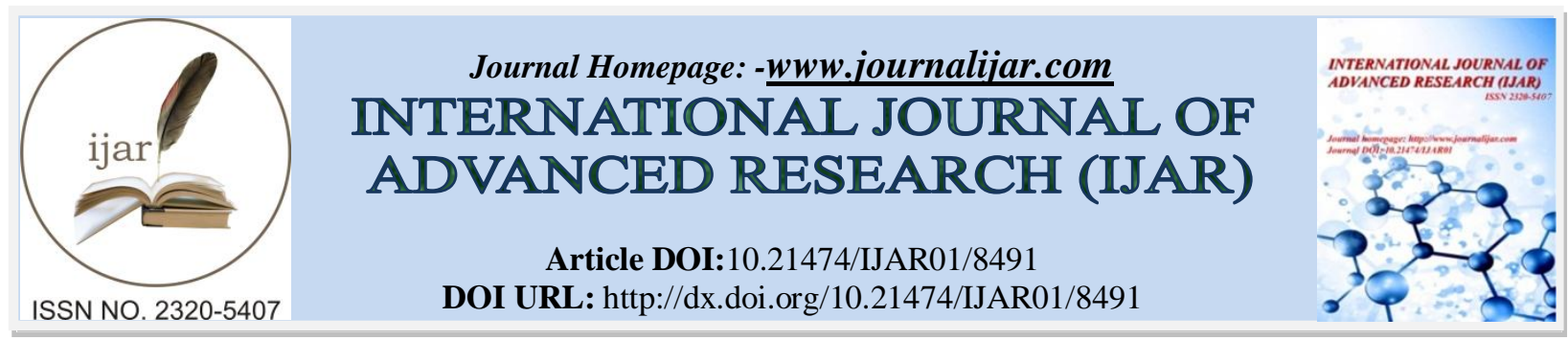

RESEARCH ARTICLE

\title{
IMMEDIATE EFFECT OF MOBILIZATION ON PAIN AND OVERHEAD REACH INPATIENTS WITH SHOULDER IMPINGEMENT SYNDROME.
}

\author{
Ibrahim A. Alwesaly ${ }^{1,2}$ and Mohammed S. Abdelsalam ${ }^{2,3}$. \\ 1. Aramco John Hopkins hospital, Dammam, KSA. \\ 2. Department of Physical Therapy, College of Applied Medical Sciences, Imam Abdulrahman Bin Faisal \\ University, Dammam, KSA. \\ 3. Department of Physical Therapy for Musculoskeletal disorders and its Surgery, Faculty of Physical Therapy, \\ Cairo University.
}

\section{Manuscript Info \\ Manuscript History \\ Received: 04 December 2018 \\ Final Accepted: 06 January 2019 \\ Published: February 2019}

Key words:-

Mobilization, Shoulder impingement syndrome, Shoulder pain, Overhead reach.

\begin{abstract}
Therapists using mobilization techniques commonly implement a prepost session (application) evaluation of symptoms. Symptoms usually checked are pain intensity and range of motion. This suggests an immediate effect of the applications. Shoulder impingement syndrome (SIS) is a common cause of shoulder pain and commonly shows restriction in shoulder range of motion, especially shoulder elevation. In this study, the aim was to evaluate the immediate effects of shoulder mobilization on pain and overhead reach in patients with SIS. Methods: 50 patients with SIS were recruited and randomly assigned to mobilization and control groups. Patients in both groups received a single session. In mobilization group, they received transcutaneous electrical nerve stimulation (TENS) and mobilization techniques, while control group received TENS only. Pain intensity and overhead reach distance (ORD) were evaluated pre and post session. Results showed significantly decreased pain intensity and increased ORD in patients of mobilization group. Patients in the control group showed significant decreased pain intensity, however, it was significantly less than the pain reduction achieved in mobilization group. Conclusion:according to the findings, mobilization showed significant immediate effect on pain and ORD in patients with SIS, which supports the practice of pre- post session evaluation used in mobilization.
\end{abstract}

Copy Right, IJAR, 2019,. All rights reserved.

\section{Introduction:-}

Shoulder impingement syndrome (SIS) is the leading cause of shoulder pain, as it constitutes about $44-65 \%$ of all shoulder painful conditions seeking medical help (Greenberg, 2014; Michener et al., 2003; van der Windt et al., 1995).SIS is a term that describes the 'impingement' of soft tissue structures, frequently involving rotator cuff tendons, in the subacromial space (Kachingwe et al., 2008). Rotator cuff impingement may progress to various degrees of rotator cuff injuries if not properly managed (Khan et al., 2013). SIS is commonly associated with pain, tenderness and limited shoulder range of motion (Millar et al., 2006). Further, SIS was suggested to be accompanied by weakness of glenohumeral muscles as a result of rotator calf pathology or muscles inhibition due to pain

Corresponding Author:-Mohammed S. Abdelsalam.

Address:-Department of Physical Therapy for Musculoskeletal disorders and its Surgery, Faculty of 321 Physical Therapy, Cairo University. 
(Hengeveld E and Banks K, 2005). A wide range of conservative treatment options are in use to manage SIS in general practice, including relative rest, non-steroidal anti-inflammatory medications, corticosteroid injections, exercise, transcutaneous electrical nerve stimulation (TENS) (Hansson and Lundeberg, 1999), and manual therapy (Dorrestijn et al., 2007).

Passive joints mobilization is a form of manual therapy that can reduce shoulder pain, improve range of motion (ROM), and improve function as well (Ho et al., 2009). The assessment process is a part of all manual therapy approaches, whether articular or soft tissue oriented. As a common concept in different approaches, initial session assessments include initial pre-intervention, and first post-intervention re-examination (Cook, 2007; Hengeveld E and Banks K, 2005). Immediate re-examination following each intervention session is supposed to show the effects of the applied treatments or treatment outcomes (Cook, 2007). Accordingly, studying the effects following application of manual therapy techniques might provide evidence to support, or else discourage, this practice. Immediate effects of initial manual therapy sessions in shoulder pain and dysfunction had been studied in some previous studies (Godges et al., 2003; Hsu et al., 2000; Strunce et al., 2009; Teys et al., 2008; Wang and Meadows, 2010; Yu et al., 2015). They included the immediate effects of soft tissue mobilization (Godges et al., 2003), mobilization with movement (Teys et al., 2008), remote effects of cervical (Wang and Meadows, 2010) and thoracic (Strunce et al., 2009) mobilizations on shoulder pain and dysfunction, besides passive joint mobilization (Hsu et al., 2000; Yu et al., 2015). While Hsu et al. (2000) compared the effects of anterior posterior glenohumeral glides at mid- range and end range mobilizations on cadavers on shoulder abduction range of motion, Yu et al. (2015) used end range anterior-posterior glide to check for glenohumeral internal rotation range of motion and skin temperature changes. To the best knowledge of the authors, the immediate effects of shoulder passive accessory movements mobilization techniques have not been reported for pain and functional overhead reach in SIS. Therefore, the aim of the current study is to examine the immediate effects of shoulder passive mobilization techniques on patients' pain, overhead reach in patients with SIS. The hypotheses were that there would not be any significant difference in pain between pre- and post- session evaluations in either Mobilization or control groups. Moreover, there will be no significant difference in overhead reach distance in the involved (evaluated pre and post session) and sound (evaluated at baseline) shoulders in SIS patients in either Mobilization or control groups.

\section{Materials and Methods:-}

The current design is a two groups' pretest- posttest study. Where 50 male patient age ranged between 24-40 years, suffering unilateral SIS participated. Sample size calculation was done using a G*power software at a power of 0.90 . Patients were randomly assigned either to mobilization or control groups. Random assignment to groups was based on patients' order of recruitment. Prior to patients' recruitment, a random number generator was used to create a set of random numbers from 1-50, without repetition, representing the recruitment order of patients. The number of even values were assigned to mobilization group, while odd recruitment numbers were assigned to control group. All patients were recruited from the outpatient clinics of security forces hospital (SFH), Dammam, Saudi Arabia. Patients were recruited to the study if they were referred to physical therapy department diagnosed as unilateral SIS patients. The referring orthopedists were asked to check the clinical findings prior to inclusion. Patients were included if symptoms were continuous for at least one month prior to participation. In addition, clinical findings included pain in the proximal region of the armat the shoulder area. Further, one positive test of the Neer's, Hawkins-Kennedy impingement tests, and painful arc during shoulder abduction and/or flexion (Kromer et al., 2013). Patients were excluded from the study if they were diagnosed to sustain a complete rotator cuff tear, or frozen shoulder. Also, if they had an intra-articular cortisone injection within the last 3 months prior to participation, they sustained previous injuries, deformities or performed surgeries of the cervical spine, shoulder or shoulder girdle, or they could not assume the proper starting position for shoulder mobilization technique applied in this study $\left(90^{\circ}\right.$ shoulder abduction, or higher, in frontal plane). Patients who met the inclusion/exclusion criteria were interviewed prior to participation. Patients received complete explanation of the objectives of the study, evaluation and intervention procedures pertinent to their group, as well as the average duration of the intervention session. Afterwards, patients were asked to sign an informed consent form. The study received ethical approval of the institutional research board (IRB) of Imam Abdulrahman Bin Faisal University, Dammam, Saudi Arabia.

\section{Evaluation procedures}

The same examiner who was blinded to patients group allocation evaluated patients in both groups. All patients undertook the same evaluation procedures pre- and post- session for affected shoulders. Evaluation included pain intensity using visual analog scale (VAS), and ORD using overhead stand reach test (OSRT). On the other hand, 
unaffected shoulders of patients in both groups were evaluated pre- session for comparison of overhead reach distance (ORD).

\section{Pain}

Patients were asked to assess their pain intensity on VAS; a horizontal $100 \mathrm{~mm}$ line denoted at one end by "no pain" and the other end "Intolerable pain"(Ferreira-Valente et al., 2011). Patients were asked to mark the severity of their pain the way they felt it prior to session and at the end of session. The distance each patient marked was measured from the "no pain" end to indicate the pain intensity.

\section{Overhead stand reach test (OSRT)}

Patients standing head height were measured on a vertical wall mounted scale. Then patients were asked to face the wall and reach overhead as high as they could. The difference between the patients' maximum overhead reach and standing head heights in centimeters were recorded for three trials and averages were used for analysis (Hayes et al., 2001).

\section{Treatment procedure}

Patients in both the mobilization and control groups received TENS (Sonopuls 692, ENRAF-NONIUS, Rotterdam, Netherlands). TENS was applied using one channel (2 electrodes) position on the anterior and posterior aspects of the shoulder respectively with the patient's arm rested beside the body while patient assumed supine lying position. The TENS device was adjusted to a frequency of $100 \mathrm{~Hz}, 15 \mathrm{~mA}, 150 \mu \mathrm{s}$, and a time of 20 minutes (Korkmaz et al., 2010)

\section{Mobilization}

Patients in Mobilization group received passive joint distraction and glide mobilization oscillatory techniques of the affected shoulders. Therapist started with lateral glenohumeral distraction. Application of lateral distraction was from a position of $55^{\circ}$ abduction and $30^{\circ}$ horizontal abduction, and arm $25^{\circ}$ externally rotated in the horizontal plane. The therapist stabilized the elbow by his distal hand and laterally distracted the head of humerus from the plane of glenoid cavity by the proximal hand fitted as close as possible to the axilla. The application was 2-3 oscillations per second for 2 minutes, applying grade II oscillatory mobilization (Kisner and Colby, 2007). Anterior- posterior glenohumeral glide was done from $90^{\circ}$ shoulder abduction and elbow flexion. Therapist held distal humerus and supported patient's forearm by his distal hand and forearm. Therapist's proximal arm gave the anterior- posterior mobilization force at the anterior part of the head of humerus, as close to the glenoid cavity as possible. Then therapist, applied inferior glide from $90^{\circ}$ shoulder abduction or higher, according to patient's available range of shoulder abduction. For inferior glide, therapist stood beside patient's head and supported the abducted patient's arm by the distal hand and forearm. Therapist cupped the proximal most part of the humerus head, as close to glenoid cavity as possible by the $1^{\text {st }}$ web space of the treating (proximal) hand. Then therapist provided the inferior directed mobilization force. Grade III oscillatory glides were applied using smooth movements at approximate rate of 2-3 repetitions per second for 2 minutes for each gliding direction(Hengeveld E and Banks K, 2005; Kisner and Colby, 2007).

\section{Statistical Analysis}

Data were analyzed using statistical package SPSS version 20. Demographic data for patients in both groups were compared using independent sample t- test. Test of normality were conducted on the outcome parameters using Shapiro-Wilk test. Further, each of the outcomes of pain and SRDwithin and between groups were compared using a mixed model ANOVA. P value set to $<0.05$.

\section{Results:-}

Demographic data of patients in Mobilization and control groups are presented and compared in table (1).

Table 1:-Demographic data of patients in Mobilization and control groups.

\begin{tabular}{|l|l|l|l|l|}
\hline & Groups & \multicolumn{2}{l|}{ T-test } \\
\hline & $\begin{array}{l}\text { Mobilization group } \\
\text { Mean }( \pm \text { SD) }\end{array}$ & $\begin{array}{l}\text { Control group } \\
\text { Mean }( \pm \text { SD) }\end{array}$ & T- Stat. & Sig. \\
\hline Age (years) & $36.68( \pm 4.27)$ & $35.48( \pm 5.17)$ & 0.894 & 0.376 \\
\hline Weight $(\mathrm{kg})$ & $88.72( \pm 16.29)$ & $84.78( \pm 11.46)$ & 0.988 & 0.328 \\
\hline
\end{tabular}




\begin{tabular}{|l|l|l|l|l|}
\hline Height $(\mathrm{cm})$ & $170.98(+5.49)$ & $172.84(+4.99)$ & -1.253 & 0.216 \\
\hline BMI $\left(\mathrm{kg} / \mathrm{m}^{2}\right)$ & $30.26(\underline{4.86)}$ & $28.39(\underline{ \pm 3} .77)$ & 1.518 & 0.136 \\
\hline
\end{tabular}

$\mathbf{P}<0.05$

\section{Outcome parameters}

According to normality test, pain outcomes pre and post session in either groups were normally distributed. Likewise, ORD outcomes pre and post session of affected shoulders and baseline of sound shoulders in either groups were normally distributed. Accordingly, parametric statistics were used.

\section{Pain (VAS)}

Mixed model ANOVA showed significant main effect of pain over evaluations $\left(F_{(1,48)}=88.075\right.$, $\left.p<0.000\right)$, as well as pain-groups interaction $\left(\mathrm{F}_{(1,48)}=25.693, \mathrm{p}<0.000\right)$. Pairwise comparisons of pre and post session evaluations within mobilization and control groups showed significantly lower post session compared to pre session mean VAS values in both groups. Between groups, comparisons of mean VAS were non- significantly different at pre- session evaluation, while mean VAS values showed significantly lower VAS in mobilization compared to control group (Table 2)

Table 2:-Comparison of mean pain intensities between patients in mobilization and control groups at pre and post session evaluations.

\begin{tabular}{|c|c|c|c|c|}
\hline & & & Pairwise & arisons \\
\hline Evaluations & $\begin{array}{l}\text { Mobilization } \\
\text { Mean }(+ \text { SD })\end{array}$ & $\begin{array}{l}\text { Control } \\
\text { Mean }( \pm \mathrm{SD})\end{array}$ & F- Stat. & Sig. \\
\hline Pre- session & $4.34( \pm 1.27)$ & $4.80( \pm 1.78)$ & 1.110 & 0.297 \\
\hline Post- session & $2.62(+1.26)$ & $4.29(+1.96)$ & 12.836 & $0.001 *$ \\
\hline $\begin{array}{l}\text { Pairwise comparisons } \text { F-stat } \\
\text { Sig. }\end{array}$ & $\begin{array}{c}104.454 \\
0.000^{*}\end{array}$ & $\begin{array}{l}9.314 \\
0.004 *\end{array}$ & & \\
\hline
\end{tabular}

$\mathrm{P}<0.05$

\section{Overhead reach distance (ORD)}

Mixed model ANOVA showed significant main effect of overhead reach over evaluations $\left(\mathrm{F}_{(2,47)}=68.211, \mathrm{p}<\right.$ $0.000)$, as well as pain-groups interaction $\left(\mathrm{F}_{(2,47)}=57.957, \mathrm{p}<0.000\right)$. Pairwise comparisons of pre and post session evaluations of affected shoulders within mobilization group showed significantly higher ORD post session compared to pre session. Moreover, pre session ORD was significantly lower in the affected shoulder compared to sound shoulder, but there was no significant difference ORD measured post session compared to sound shoulder. Within the control group, pairwise comparisons showed non- significant difference between pre and post session ORD. Further, in control group ORD of affected shoulder at either pre or post session evaluations were significantly lower than ORD of their sound shoulders. Between groups comparisons showed non- significant differences between ORD in patients of both groups at pre session evaluations of the affected shoulders, as well as the sound shoulders. Post session, the ORD of affected shoulders of patients in mobilization group was significantly higher than that in the control group (Table 3).

Table 3:-Comparison of mean overhead reach distance (ORD) between patients in mobilization and control groups at pre and post session evaluations .

\begin{tabular}{|ll|l|l|l|l|l|}
\hline & & \multicolumn{2}{l|}{ Groups } & \multicolumn{2}{l|}{ Pairwise comparisons } \\
\hline & & & $\begin{array}{l}\text { Mobilization } \\
\text { Mean }( \pm \text { SD })\end{array}$ & $\begin{array}{l}\text { Control } \\
\text { Mean }( \pm \text { SD })\end{array}$ & F- Stat. & Sig. \\
\hline affected (pre session) & $(1)$ & & $46.66( \pm 4.51)$ & $43.84( \pm 5.64)$ & 3.830 & 0.056 \\
\hline Affected (post session) & $(2)$ & & $50.60( \pm 3.94)$ & $44.46( \pm 5.42)$ & 21.071 & $0.000^{*}$ \\
\hline Sound & $(3)$ & & $50.85( \pm 3.72)$ & $50.29( \pm 3.91)$ & 0.260 & 0.612 \\
\hline Pairwise comparisons & F-stat & & 52.499 & 38.944 & & \\
Paired Sig. & $(1-2)$ & $0.000^{*}$ & 0.110 & & \\
& $(1-3)$ & $0.000^{*}$ & $0.000^{*}$ & & \\
& $(2-3)$ & 0.711 & $0.000^{*}$ & & \\
\hline
\end{tabular}

$\mathrm{P}<0.05$ 


\section{Discussion:-}

This study investigated the immediate effects of shoulder mobilization on pain, and overhead reach distance (ORD) in patients with SIS.

In this study, there was an immediate pain reduction in both mobilization and control groups post-session. However, pain intensity post-session was significantly lower in mobilization group compared to control group. It worth notice that mean pain reduction in mobilization group was $1.72 \mathrm{~cm}$ which exceeded the minimum clinically important difference (MCID) which was predicted in earlier studied to be $1.4 \mathrm{~cm}$ change in mean VAS scores (Kelly, 2001; Tashjian et al., 2009; Tashjian et al., 2017). However, a limitation in this study based on the effect of TENS in the control group $(0.51 \mathrm{~cm}$ post-session mean pain reduction) was that results could not be completely attributed to mobilization.

Some mechanisms could explain how mobilization techniques used might have contributed to pain reduction in mobilization group in the current study. One mechanism could be stimulation of joint mechanoreceptors, which have been suggested to block afferent pain signals and thus reduce pain (Do Moon et al., 2015). Another mechanism may be the inhibitory effect that the stimulation of joint mechanoreceptors has on tension of the surrounding muscles. Reduced reflex muscle contraction around a joint was suggested to reduce articular pain via the dispersal of muscle waste metabolites that irritate the joint and accumulate due to muscle ischemia (Zusman, 1986). The stimulation of periaqueductal grey matter (PGM) and release of endogenous analgesia had been suggested as a mechanism of initial pain reduction when applying manual therapy (Bialosky et al., 2009; Voogt et al., 2015; Wright, 1995).

To the best of our knowledge, there is no previous investigation of the immediate effects of mobilization using joint gliding on shoulder pain. One a previous study, authors examined the immediate effect of mobilization with movement (MWM) on shoulder pain (Teys et al., 2008). A basic difference between the current study and that previous study is the difference in the mobilization techniques. Further, pain intensity in the current study was assessed using VAS while in the previous study they used pain pressure threshold (PPT). Despite the differences, both studies pointed out that the applications under investigation improved post session compared to pre session pain. Despite there was no measurement pre and post session (only pre and post all 10 sessions), and there was a different pathology the study of Nicholson (1985) sounds to support the current findings. Where, Nicholson (1985) examined the effects of passive shoulder joint mobilization on pain and ROM of shoulder joint in adhesive capsulitis. There was a self- reported symptoms improvement following each session of the 10 sessions of the study. Furthermore, the immediate remote effects of cervical spine (McClatchie et al., 2009), and thoracic spine and ribs (Strunce et al., 2009) mobilization on shoulder pain have been investigated. Results from these studies demonstrated significant improvement of shoulder pain immediately following the application of spine mobilization techniques. However, it is unlikely to consider these results as completely conforming with the present study due to differences in the regions of application of mobilization techniques; therefore, the possible underlying mechanisms to explain the results are likely different.

The results of the present study that showed significant pain reduction in control group are comparable and similar finding to results of a previous investigation on TENS in the management of shoulder pain in patients with SIS Stage I (Başkurt et al., 2006). Alike the present study, their results showed that a single session can reduce pain (Başkurt et al., 2006). A similar study investigated the effects of a single session of TENS, compared to a sham TENS application, on functional magnetic resonance imaging (FMRI) of a group of regions of interest (ROI) in patients with subacromial impingement. Their results showed a significant reduction of pain; and, most interestingly, this was correlated with the modulation of FMRI activity in the ROI (Kocyigit et al., 2012). In terms of pain inhibition, the gate control theory of pain is one of the most widely used theories for explaining the effect of TENS on pain inhibition (Sluka and Walsh, 2003). The release of endogenous opioids in response to TENS stimulation has been considered an alternative explanation for the induced hypoalgesic effect of TENS in painful conditions (Kalra et al., 2001; Sluka and Walsh, 2003).

\section{Overhead reach distance}

The results of the current study showed significant improvement of the ORD in mobilization group, as well as between mobilization and control groups. Patients with SIS showed abnormal minor, yet significant, anterior translation of the head of humerus during shoulder abduction in the scapular plane (Tyler et al., 2010). It was reported earlier that anterior translation was marked in early flexion movement in the scapular plane $\left(30^{\circ-} 60^{\circ}\right)$, which was then followed by a reduction in the posterior translation of the humeral head through the $60^{\circ}$ to $120^{\circ}$ 
range (Lin et al., 2008; Yu et al., 2015). Accordingly, improvement of ORD may be explained by adjustment of humeral translation, which relieves posterior capsular tightness in patients with SIS (Tyler et al., 2010). Mobilization was reported to improve the flexibility of the joint capsule, normalize the kinematics of the glenohumeral joint, and restore the normal glide (translation) of the humeral head (Lin et al., 2008; Yu et al., 2015).

Improved overhead reach with mobilization was reported in a previous study, where shoulder mobilization of Grades I-IV was applied in conjunction with exercise for six sessions over three weeks (Bang and Deyle, 2000). These results tend to support results in the current study; except that in the current study, gliding techniques were applied for one session. In addition, mobilization in the present study was applied with TENS, not with exercise. A study was conducted to check the immediate effect of one session of soft tissue mobilization of the subscapularis muscle and proprioceptive neuromuscular (PNF) training for the subscapularis muscle in the study group versus the control group (Godges et al., 2003). Their results showed that ORD was significantly improved in the experimental group compared to the control group. This study is similar to the current study in its assessment of the immediate effects of a manual therapy technique on shoulder ROM and functional overhead reach; their results may also support our results to some extent. Both studies provide clues that immediate overhead distance changes may occur as tight, soft tissues around the shoulder are released. Another study that dealt with the immediate effects of soft tissue mobilization and PNF in overhead shoulder reach was conducted on patients with impingement syndrome (Al Dajah, 2014). Results showed significant improvement in ORD immediately following soft tissue mobilization and PNF intervention compared to therapeutic ultrasounds applied to patients in the other group. Once more, these results could be considered similar to our results, despite differences in the manual therapy technique used and the target structure (subscapularis muscle).

A previous study comparing the effect of combined passive shoulder mobilization and physical therapy to physical therapy alone in nine sessions was conducted over three weeks (Conroy and Hayes, 1998). One of their outcome measures was functional overhead reach to $135^{0}$ shoulder flexion. They reported improvement in both groups, but there was no significant difference between the groups. Their results contradict the results of the current study. Several reasons may explain the source of difference in the results, the most significant of which is the inclusion of other therapeutic exercises and techniques, such as self-stretching and soft tissue mobilization, which might affect overhead shoulder reach in their control group.

A limitation of the current study was the participation of male patients only. This limits the study outcomes to the male gender. Would the outcomes had been affected if female patients participated is not clear.

\section{Conclusion:-}

The aim of this study was to investigate the immediate effect of shoulder mobilization on pain, and ORD. Results pointed out that shoulder mobilization, using joint distraction and gliding, has an immediate effect on reducing pain intensity and increasing ORD in patients with SIS. Accordingly, within the conditions applied to this study, it supported the pre- post session comparison paradigm used in evaluation of mobilization effects.

\section{Conflict of Interest}

The authors declare that they have no conflict of interest.

\section{References:-}

1. Al Dajah, S. B. (2014). Soft Tissue Mobilization and PNF Improve Range of Motion and Minimize Pain Level in Shoulder Impingement. J PhysTherSci, 26(11), 1803-1805.

2. Bang, M. D. and Deyle, G. D. (2000). Comparison of supervised exercise with and without manual physical therapy for patients with shoulder impingement syndrome. J Orthop Sports PhysTher, 30(3), 126-137.

3. Başkurt, Z., Başkurt, F., Özcan, A. and Yilmaz, Ö. (2006). The immediate effects of heat and TENS on pressure pain threshold and pain intensity in patients with Stage I shoulder impingement syndrome. Pain Clinic, 18(1), 81-85. doi: 10.1163/156856906775249839

4. Bialosky, J. E., Bishop, M. D., Price, D. D., Robinson, M. E. and George, S. Z. (2009). The mechanisms of manual therapy in the treatment of musculoskeletal pain: a comprehensive model. Man Ther, 14(5), 531-538.

5. Conroy, D. E. and Hayes, K. W. (1998). The effect of joint mobilization as a component of comprehensive treatment for primary shoulder impingement syndrome. J Orthop Sports PhysTher, 28(1), 3-14.

6. Cook, C. (2007). Orthopedic manual therapy: an evidence based approach. Upper Saddle River, N.J: Pearson/Prentice Hall. 
7. Do Moon, G., Lim, J. Y., Kim da, Y. and Kim, T. H. (2015). Comparison of Maitland and Kaltenborn mobilization techniques for improving shoulder pain and range of motion in frozen shoulders. J PhysTherSci, 27(5), 1391-1395.

8. Dorrestijn, O., Stevens, M., Diercks, R. L., van der Meer, K. and Winters, J. C. (2007). A new interdisciplinary treatment strategy versus usual medical care for the treatment of subacromial impingement syndrome: a randomized controlled trial. BMC Musculoskeletal Disorders, 8, 15-15. doi: 10.1186/1471-2474-8-15

9. Ferreira-Valente, M. A., Pais-Ribeiro, J. L. and Jensen, M. P. (2011). Validity of four pain intensity rating scales. Pain, 152(10), 2399-2404.

10. Godges, J. J., Mattson-Bell, M., Thorpe, D. and Shah, D. (2003). The immediate effects of soft tissue mobilization with proprioceptive neuromuscular facilitation on glenohumeral external rotation and overhead reach. J Orthop Sports PhysTher, 33(12), 713-718.

11. Greenberg, D. L. (2014). Evaluation and treatment of shoulder pain. Med Clin North Am, 98(3), 487-504.

12. Hansson, P. and Lundeberg, T. (1999). Transcutaneous electrical nerve stimulation, vibration and acupuncture as pain-relieving measures. Textbook of pain, 1341-1351.

13. Hayes, K., Walton, J. R., Szomor, Z. R. and Murrell, G. A. (2001). Reliability of five methods for assessing shoulder range of motion. Aust J Physiother, 47(4), 289-294.

14. Hengeveld E and Banks K. (2005). Maitland's Peripheral Manipulation (4th edition ed.): ButterworthHeinemann.

15. Ho, C. Y., Sole, G. and Munn, J. (2009). The effectiveness of manual therapy in the management of musculoskeletal disorders of the shoulder: a systematic review. Man Ther, 14(5), 463-474.

16. Hsu, A. T., Ho, L., Ho, S. and Hedman, T. (2000). Joint position during anterior-posterior glide mobilization: its effect on glenohumeral abduction range of motion. Arch Phys Med Rehabil, 81(2), 210-214.

17. Kachingwe, A. F., Phillips, B., Sletten, E. and Plunkett, S. W. (2008). Comparison of manual therapy techniques with therapeutic exercise in the treatment of shoulder impingement: a randomized controlled pilot clinical trial. J Man ManipTher, 16(4), 238-247.

18. Kalra, A., Urban, M. O. and Sluka, K. A. (2001). Blockade of opioid receptors in rostral ventral medulla prevents antihyperalgesia produced by transcutaneous electrical nerve stimulation (TENS). J PharmacolExpTher, 298(1), 257-263.

19. Kelly, A. M. (2001). The minimum clinically significant difference in visual analogue scale pain score does not differ with severity of pain. Emerg Med J, 18(3), 205-207.

20. Khan, Y., Nagy, M. T., Malal, J. and Waseem, M. (2013). The painful shoulder: shoulder impingement syndrome. Open Orthop J, 7, 347-351.

21. Kisner, C. and Colby, L. A. (2007). Therapeutic exercise: foundations and techniques (5th edition ed.). philadelphia: FA Davis.

22. Kocyigit, F., Akalin, E., Gezer, N. S., Orbay, O., Kocyigit, A. and Ada, E. (2012). Functional magnetic resonance imaging of the effects of low-frequency transcutaneous electrical nerve stimulation on central pain modulation: a double-blind, placebo-controlled trial. Clin J Pain, 28(7), 581-588.

23. Korkmaz, O. K., Capaci, K., Eyigor, C. and Eyigor, S. (2010). Pulsed radiofrequency versus conventional transcutaneous electrical nerve stimulation in painful shoulder: a prospective, randomized study. Clinical Rehabilitation, 24(11), 1000-1008. doi: http://dx.doi.org/10.1177/0269215510371417

24. Kromer, T. O., de Bie, R. A. and Bastiaenen, C. H. (2013). Physiotherapy in patients with clinical signs of shoulder impingement syndrome: a randomized controlled trial. J Rehabil Med, 45(5), 488-497.

25. Lin, H. T., Hsu, A. T., An, K. N., Chang Chien, J. R., Kuan, T. S. and Chang, G. L. (2008). Reliability of stiffness measured in glenohumeral joint and its application to assess the effect of end-range mobilization in subjects with adhesive capsulitis. Man Ther, 13(4), 307-316.

26. McClatchie, L., Laprade, J., Martin, S., Jaglal, S. B., Richardson, D. and Agur, A. (2009). Mobilizations of the asymptomatic cervical spine can reduce signs of shoulder dysfunction in adults. Man Ther, 14(4), 369-374.

27. Michener, L. A., McClure, P. W. and Karduna, A. R. (2003). Anatomical and biomechanical mechanisms of subacromial impingement syndrome. ClinBiomech (Bristol, Avon), 18. doi: 10.1016/s0268-0033(03)00047-0

28. Millar, A. L., Jasheway, P. A., Eaton, W. and Christensen, F. (2006). A retrospective, descriptive study of shoulder outcomes in outpatient physical therapy. Journal of Orthopaedic\& Sports Physical Therapy, 36(6), 403-414.

29. Nicholson, G. G. (1985). The effects of passive joint mobilization on pain and hypomobility associated with adhesive capsulitis of the shoulder. J Orthop Sports PhysTher, 6(4), 238-246.

30. Sluka, K. A. and Walsh, D. (2003). Transcutaneous electrical nerve stimulation: Basic science mechanisms and clinical effectiveness (Vol. 4, pp. 109-121). United States: Elsevier Inc. 
31. Strunce, J. B., Walker, M. J., Boyles, R. E. and Young, B. A. (2009). The immediate effects of thoracic spine and rib manipulation on subjects with primary complaints of shoulder pain. J Man ManipTher, 17(4), 230-236.

32. Tashjian, R. Z., Deloach, J., Porucznik, C. A. and Powell, A. P. (2009). Minimal clinically important differences (MCID) and patient acceptable symptomatic state (PASS) for visual analog scales (VAS) measuring pain in patients treated for rotator cuff disease. J Shoulder Elbow Surg, 18(6), 927-932.

33. Tashjian, R. Z., Hung, M., Keener, J. D., Bowen, R. C., McAllister, J., Chen, W., Ebersole, G., Granger, E. K. and Chamberlain, A. M. (2017). Determining the minimal clinically important difference for the American Shoulder and Elbow Surgeons score, Simple Shoulder Test, and visual analog scale (VAS) measuring pain after shoulder arthroplasty. J Shoulder Elbow Surg, 26(1), 144-148.

34. Teys, P., Bisset, L. and Vicenzino, B. (2008). The initial effects of a Mulligan's mobilization with movement technique on range of movement and pressure pain threshold in pain-limited shoulders. Man Ther, 13(1), 37-42.

35. Tyler, T. F., Nicholas, S. J., Lee, S. J., Mullaney, M. and McHugh, M. P. (2010). Correction of posterior shoulder tightness is associated with symptom resolution in patients with internal impingement. Am J Sports Med, 38(1), 114-119.

36. van der Windt, D. A., Koes, B. W., de Jong, B. A. and Bouter, L. M. (1995). Shoulder disorders in general practice: incidence, patient characteristics, and management. Annals of the Rheumatic Diseases, 54(12), 959964.

37. Voogt, L., de Vries, J., Meeus, M., Struyf, F., Meuffels, D. and Nijs, J. (2015). Analgesic effects of manual therapy in patients with musculoskeletal pain: a systematic review. Man Ther, 20(2), 250-256.

38. Wang, S. S. and Meadows, J. (2010). Immediate and carryover changes of C5-6 joint mobilization on shoulder external rotator muscle strength. J Manipulative PhysiolTher, 33(2), 102-108.

39. Wright, A. (1995). Hypoalgesia post-manipulative therapy: a review of a potential neurophysiological mechanism. Man Ther, 1(1), 11-16.

40. Yu, I. Y., Jung, I. G., Kang, M. H., Lee, D. K. and Oh, J. S. (2015). Immediate effects of an end-range mobilization technique on shoulder range of motion and skin temperature in individuals with posterior shoulder tightness. J PhysTherSci, 27(6), 1723-1725.

41. Zusman, M. (1986). Spinal manipulative therapy: review of some proposed mechanisms, and a new hypothesis. Aust J Physiother, 32(2), 89-99. 